\title{
Evaluación de pavimentos y decisiones de conservación con base en sistemas de inferencia difusos
}

\author{
Pavement Evaluation and Maintenance Decisions Based on Fuzzy Inference \\ Systems
}

\author{
Beltrán-Calvo Gloria Inés \\ Facultad de Ingeniería \\ Universidad Nacional de Colombia \\ Correos:gibeltranc@unal.edu.co; gbeltranc@iingen.unam.mx.
}

\author{
Romo-Organista Miguel Pedro \\ Instituto de Ingeniería \\ Universidad Nacional Autónoma de México \\ Correo:mromo@pumas.iingen.unam.mx.
}

Información del artículo: recibido: noviembre de 2012, aceptado: marzo de 2013

\section{Resumen}

Las decisiones de conservación y la vida remanente de pavimentos en servicio, dependen en buena parte de la condición estructural que exhiben durante su operación. Con el ánimo de proveer una metodología que permita caracterizar el pavimento de manera rápida, con los niveles de detalle que se deseen, en este trabajo se plantean tres sistemas basados en la lógica difusa, para inferir la condición de rigidez y deterioro, así como las decisiones de conservación. Para esos fines, se utilizaron datos de auscultación no destructiva registrados en sistemas de pavimento de tres y cuatro capas, incluyendo estructuras tradicionales y estructuras invertidas (con capa de sub-base rigidizada). Las bases de datos incluyen información sobre magnitud y severidad de daños estructurales y parámetros e indicadores de rigidez, derivados de pruebas de deflexión por impacto. Los sistemas desarrollados, permitieron integrar directamente en la caracterización estructural de los pavimentos, tanto el conocimiento y criterios de expertos, como las variables de carácter subjetivo y cualitativo, que se utilizan comúnmente para describir los niveles de severidad del deterioro. Asimismo, permitieron expresar los resultados mediante palabras o adjetivos cuantificadores del lenguaje, facilitando su interpretación y comprensión. En virtud de los resultados alcanzados, resulta razonable concluir que los sistemas propuestos pueden utilizarse con confianza para evaluaciones rápidas y toma de decisiones a nivel global o detallado en corredores viales.

\section{Descriptores:}

- pavimentos flexibles

- deterioro

- rigidez

- lógica difusa

- sistemas de inferencia 


\begin{abstract}
The remaining service life and maintenance decisions of existing pavements are highly dependent on stiffness and deterioration conditions throughout their operation. A non-conventional fuzzy logic based methodology is proposed in this work to address the stiffness-deterioration condition and conservation decisions, by means of three Fuzzy Inference Systems. Analysis were conducted using a database gathered from field tests performed on three and four layer pavement systems, that included traditional layer structuring and pavements having a subbase stiffer than their granular base. Information about layer thicknesses, magnitude and severity of structural distresses, and parameters and indicators derived from deflection testing by impulse load devices were used. The developed systems, allowed representing expert knowledge and linguistic variables of subjective and qualitative nature, commonly used to describe the severity levels of deterioration; thus they can be involved into structural characterization of pavements. Results were also expressed linguistically, to facilitate their interpretation and understanding. Based on the high quality of results obtained, it seems reasonable to conclude that the proposed systems could be used confidently for assessing and making decisions about pavement conservation, with low computational cost.
\end{abstract}

\section{Introducción}

Tradicionalmente, en la toma de decisiones asociadas con la conservación de pavimentos en servicio, interviene la intuición y experiencia humana, cuya subjetividad está expuesta a muchos factores de incertidumbre. Hoy en día se hace necesario acudir a técnicas y herramientas que puedan asistir y optimizar el proceso, dada la gran cantidad de factores a tener en cuenta (análisis multicriterio), la diversidad de alternativas de solución que existen, el costo de los errores que se puedan cometer y la rapidez que se requiere en las decisiones.

En el ámbito internacional, se ha mostrado cierto interés en la aplicación de metodologías no convencionales basadas en experiencias exitosas desarrolladas en el campo de la inteligencia artificial. Algunos ejemplos de ello se reportan en los estudios de Sundin y Braban (2001), Williams et al. (2004), Yella et al. (2006) y Sandra et al. (2007).

En este trabajo se abordan las herramientas de la lógica difusa para el manejo de variables subjetivas, como la condición del pavimento (mala, regular, buena) o variables lingüísticas como las recomendaciones de conservación (reconstrucción, refuerzo, etcétera). Se busca combinar las capacidades de las herramientas de cómputo y la información técnica disponible, con los recursos intelectuales y experiencia de especialistas, de modo que estos últimos queden automatizados y puedan utilizarse en la toma de decisiones de conservación en casos posteriores.
Se propone la aplicación de tres sistemas de inferencia difusos, desarrollados a partir de datos de auscultación no destructiva, para un caso de estudio seleccionado. Con ellos se pretende ofrecer una metodología capaz de inferir la condición de rigidez y deterioro, así como las acciones de conservación necesarias en sectores con deficiencia estructural; se entiende que cualquier acción que se proponga en ese sentido, mejora simultáneamente las deficiencias funcionales que puedan existir. Con el ánimo de lograr soluciones buenas, razonables y rápidas, se hace especial hincapié en el diseño y la correspondiente validación de los tres sistemas. Se espera que los resultados reflejen de manera confiable las respuestas, el comportamiento y el desempeño real del pavimento, y que además permita caracterizar rápidamente un corredor vial, con los niveles de detalle que se requieran: punto a punto o globales por sector.

\section{Fundamentos de Lógica Difusa}

La lógica no formal da cabida a conceptos poco claros o difusos de naturaleza abstracta e imprecisa para describir ciertos atributos de un sistema o fenómeno. Por ejemplo, la temperatura, si bien puede expresarse en el dominio de los números reales, es muy común expresarla con atributos como alta, media o baja. La lógica difusa (LD) provee un marco para el manejo de información cualitativa que tiene implícito algún grado de incertidumbre y subjetividad de manera que pueda formularse matemáticamente y procesarse en medios 
computacionales de forma similar al razonamiento humano.

Zadeh (1965) introdujo inicialmente el concepto de que los términos que describen atributos de una variable, pueden expresarse mediante conjuntos difusos. Cada uno de estos términos está definido por un intervalo de valores reales y cada valor tiene asociado un grado de verdad o de pertenencia a su respectivo conjunto. El grado de pertenencia a un conjunto se expresa como un continuo de números reales que pueden tomar valores entre cero y uno, creando una transición gradual entre la no pertenencia (cero) y la pertenencia total (uno).

Lo anterior se puede generalizar mediante una función de la forma $\mu_{\mathrm{mf}}(\mathrm{x})$, donde $\mu$ es el grado de pertenencia; $\mathrm{mf}$ el nombre de la función de pertenencia y x los posibles valores que toma la variable representada.

Las funciones de pertenencia pueden plantearse en forma triangular, trapezoidal o gaussiana, entre otras, y la primera es la de mayor simplicidad computacional. La selección de la mejor representación y la definición de los valores que pueden tomar las funciones de pertenencia, depende en principio de la experiencia y el conocimiento de la variable que se esté analizando.

En la figura 1 se ilustra un ejemplo de la representación difusa de la variable "\% de área afectada por grietas", con el respectivo conjunto de términos asociados; el eje horizontal representa el dominio físico de valores numéricos para las diferentes categorías de la variable.

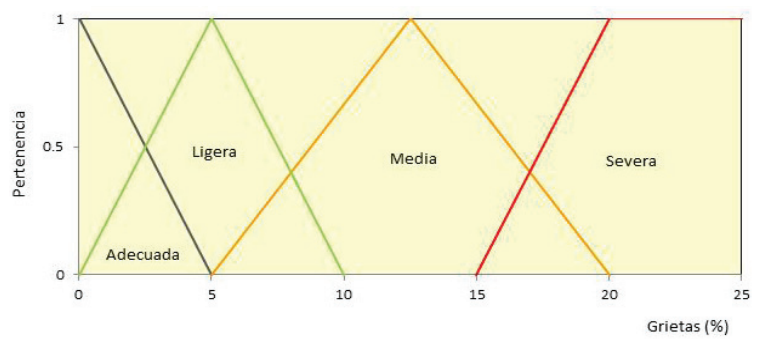

Figura 1. Representación difusa de una variable

La interpretación de las funciones de pertenencia puede describirse de la siguiente forma: cuando el área con grietas es cero, existe una pertenencia 1 o total a la condición adecuada $\left(\mu_{\text {adecuada }}(0 \%)=1\right)$. En la medida en que el área agrietada aumenta hacia 5\%, la pertenencia a la condición adecuada va disminuyendo gradualmente hasta alcanzar un valor nulo $\left(\mu_{\text {adecuada }}(5 \%)=0\right)$.

Se observa que las funciones de pertenencia pueden traslaparse de modo que, algunos valores del dominio pueden estar asociados a dos atributos diferentes, con cierto grado de pertenencia a cada uno de ellos; en esos casos, se generan condiciones interme- dias como serían, medianamente ligera y medianamente severa, entre otras.

Los conjuntos difusos pueden articularse mediante reglas de inferencia, estructuradas con premisas y consecuencias del tipo SI ... $\underline{\mathbf{Y}} / \underline{\mathbf{O}}$... ENTONCES ... (p. e. SI el agrietamiento $\mathrm{O}$ la deformación es ligera ENTONCES la condición es buena). La consecuencia de cada regla se expresa igualmente en términos difusos, cuyas categorías, funciones de pertenencia y dominio físico se deben definir previamente.

Para representar el operador lógico $\underline{\mathbf{O}}$ entre las premisas, se introduce una operación básica de unión entre conjuntos difusos, generando la agregación o implicación máxima (máx) entre ellos. Por su parte, el operador $\underline{\mathbf{Y}}$ entre dos premisas, implica la intersección entre conjuntos difusos, generando la agregación mínima (mín).

En problemas donde las soluciones dependen de muchas variables que poseen diversos atributos, se pueden plantear sistemas de inferencia difusos (SID), los cuales tienen la capacidad de procesar simultáneamente, múltiples reglas de premisas y consecuencias.

Para este trabajo, se emplea el sistema propuesto por Mandami y Assilian (1975), compuesto por:

a) la base de datos que contiene los términos asociados a las variables de entrada;

b) el grupo de reglas que articulan las variables;

c) la interfaz de "difusificación" donde cada variable de entrada y los componentes de cada regla se expresan como conjuntos difusos;

d) el mecanismo de inferencia que permite llegar a una consecuencia razonable;

e) la salida del sistema de inferencia conformada por la agregación de las consecuencias de todas las reglas que se activan simultáneamente en el sistema;

f) la interfaz de "desdifusificación" para obtener un escalar que represente el conjunto difuso en la salida.

\section{Caso aplicado}

Se seleccionó un tramo vial de $28 \mathrm{~km}$ de longitud (del K112 al K140), el cual posee los sistemas de pavimento ilustrados en la figura 2. La mayor parte del corredor posee un sistema de cuatro capas con estructura invertida, donde la sub-base presenta mayor rigidez respecto a la capa de base. En la zona intermedia existe un sistema de tres capas, donde la rigidez decrece con la profundidad. 


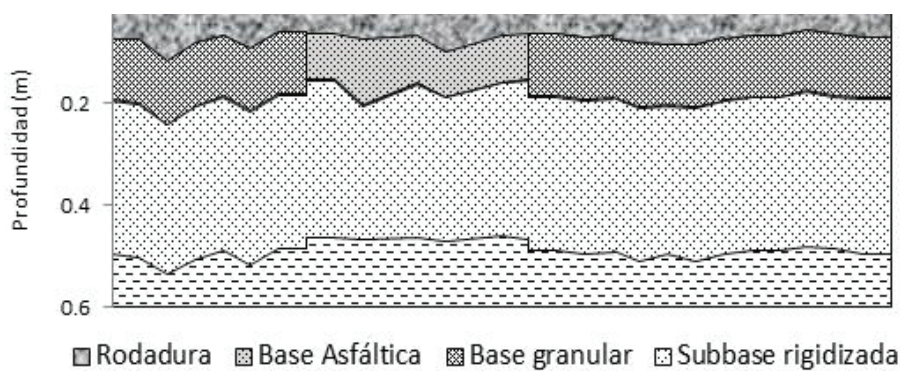

Figura 2. Sistemas estructurales en el corredor analizado
La base de datos para este estudio se conformó con información recopilada en campo mediante auscultación no destructiva (ND), incluyendo la medición del deterioro estructural y pruebas para evaluar la rigidez del pavimento.

La medición del deterioro estructural para el corredor estudiado, permitió identificar la magnitud, severidad y ubicación de los daños presentes; se dispone de registros de fenómenos de rotura, como son grietas por fatiga y baches abiertos o reparados, y registros de la profundidad de roderas o deformaciones permanentes acumuladas en las huellas de circulación de los vehículos.

En cuanto a la evaluación de la rigidez de las capas que componen el pavimento, se cuenta con registros de 280 pruebas no destructivas de deflexión por impacto. Estas pruebas intentan simular el efecto del paso de un vehículo, midiendo la respuesta de desplazamientos verticales instantáneos generados; se ha encontrado que la magnitud y forma de los desplazamientos, se encuentran fuertemente asociadas con la rigidez de las capas.

La simulación se ha logrado de manera satisfactoria con equipos de carga pesada, como el Falling Weight Deflectometer (FWD) o el Heavy Weight Deflectometer (HWD). Estos equipos aplican un impulso de carga controlada en la superficie, cuya magnitud varía entre 0.7 y 16 ton en el FWD y entre 2.7 y 24.5 ton en el HWD; la idea es reproducir la carga representativa de los vehí- culos pesados que circulan por una vía. La carga se aplica en caída libre sobre una placa circular, la cual puede ser rígida o segmentada, dependiendo del equipo empleado.

En la figura 3 se ilustra el arreglo empleado para el registro de deflexiones en el caso de estudio. Mediante 7 sensores localizados a diferentes distancias del eje de aplicación de carga, se midieron las deflexiones $\left(D_{1}\right.$, $\left.D_{2}, \ldots, D_{7}\right)$, las cuales definen el perfil de desplazamientos o cuenca de deflexión.

El propósito principal de estas pruebas es estimar los módulos elásticos de las capas de pavimento. Para el caso que nos ocupa, se cuenta con valores de módulos estimados mediante técnicas de cálculo inverso, utilizando una aproximación con redes neuronales artificiales (Beltrán y Romo, 2011).

Por otro lado, existen diversas propuestas para establecer indicadores de la capacidad estructural de un pavimento con base en las cuencas de deflexión (Barrantes et al., 2008, Horak y Emery 2006, Orozco 2005 y Zárate y Lucero 2009). Para este trabajo, se consideran la deflexión máxima, Dmáx, registrada en el sensor ubicado en el sitio de aplicación de la carga, y los índices de forma propuestos por Horak y Emery (2006), determinados mediante las siguientes expresiones, donde $\mathrm{D}_{0.33}, \mathrm{D}_{0.6} \mathrm{y}_{0.9}$ son las deflexiones medidas a $0.3,0.6$ y 0.9 $\mathrm{m}$ respectivamente del eje de carga.

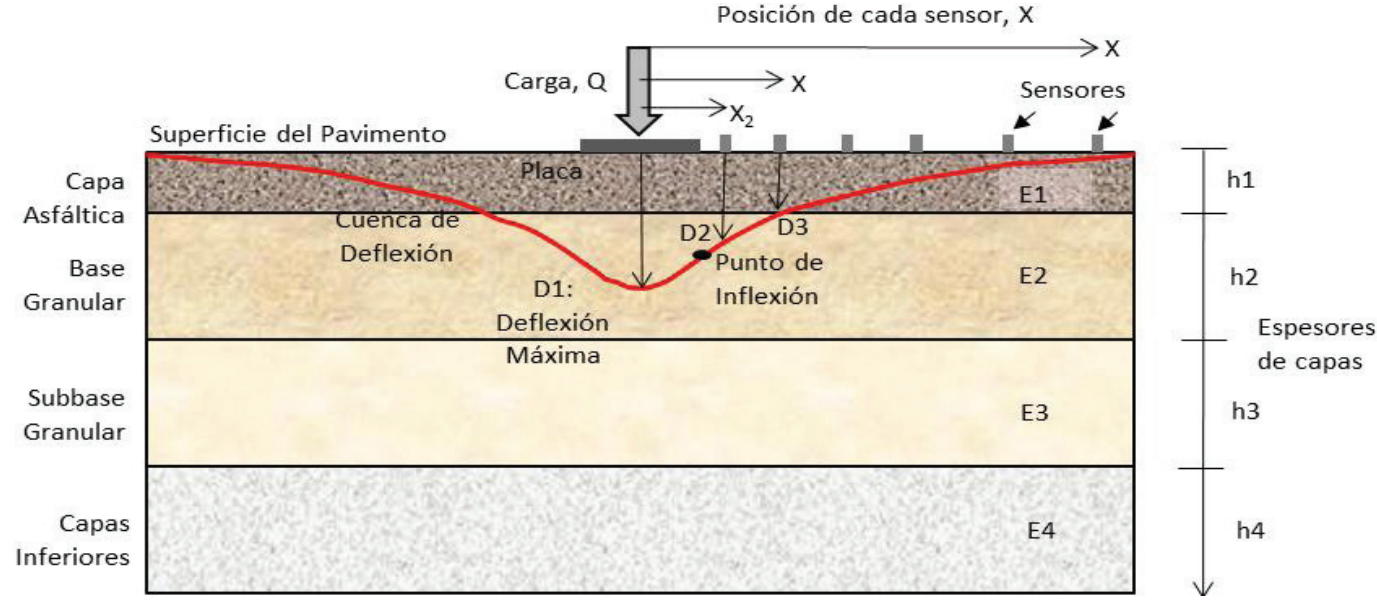

Figura 3. Prueba de deflexión por impacto y cuenca de deflexión 
- $\mathrm{BLI}=\mathrm{D}_{\text {máx }}-\mathrm{D}_{0.3}$, asociado con la rigidez de las capas de rodadura y base.

- $\mathrm{MLI}=\mathrm{D}_{0.3}-\mathrm{D}_{0.6}$, relacionado con la rigidez de la capa de sub-base.

- $\mathrm{LLI}=\mathrm{D}_{0.6}-\mathrm{D}_{0.9}$ indicador de rigidez de las capas inferiores.

\section{Planteamiento de sistemas difusos para inferir la condición y las soluciones}

Con el ánimo de caracterizar el pavimento desde los enfoques de rigidez y de deterioro estructural, así como identificar las posibles soluciones de conservación en sectores con problemas, se diseñaron tres sistemas de inferencia difusos (SID). El diseño comprende la definición de las variables de entrada-salida, su representación difusa y la definición de las reglas de inferencia que las articulan y relacionan.

Diseño del sistema para evaluar la rigidez $\left(\operatorname{SID}_{\text {rigidez }}\right)$

Las variables de entrada seleccionadas para este sistema son los módulos de capa E1, E2, E3, indicados en la figura 3, y los índices Dmáx, BLI y MLI, pues proveen conceptos que se complementan y contribuyen a identi- ficar las capas que presentan deficiencias estructurales. Para el caso de estudio, las capas inferiores reflejan alta competencia estructural; por ello, los índices y parámetros asociados a dichas capas no se consideraron para el diseño del sistema.

Los módulos de capa se discriminaron en las categorías bajo, medio y alto; la Dmáx se catalogó como buena, en riesgo, grave y severa; los índices BLI y MLI se clasificaron como bueno, en riesgo y grave. La representación difusa de cada categoría se basó en el conocimiento y criterio de expertos (ARA Inc. y ERES Consultants, 2004; Horak y Emery, 2006).

Como producto de las posibles combinaciones de variables de entrada, se establecieron categorías que califican la rigidez a la salida del sistema: pésima, mala, regular, en riesgo y adecuada; las dos primeras se dividieron en subcategorías para identificar si las deficiencias afectan la subbase (SB), la base granular (BG) o solo la capa asfáltica (CA); esto con el fin de orientar mejor las alternativas de conservación. Las categorías se asocian a una escala de calificación que varía de 0 a 10, donde la menor calificación refleja peor condición. La correspondencia entre entradas y salidas se estableció a través de un sistema de reglas de inferencia basado en los criterios mostrados en la tabla 1.

\begin{tabular}{llll}
\hline SI módulos & Y SI indicadores & Subcategoría & Rigidez \\
\hline E1 y E3 bajos & Dmax severa & CA y SB pésima & \\
E2 Y E3 bajos & MLI grave y Dmáx severa & B Y SB pésima & PésimaSB \\
E3 bajo & MLI grave y Dmáx severa & SB pésima & \\
\hline E1 y E2 bajos & BLI grave y Dmáx severa & CA y BG pésima & \multirow{2}{*}{ PésimaBG } \\
E2 bajo & BLI grave y Dmáx severa & BG pésima & \\
\hline E1 bajo & BLI grave y Dmáx severa & CA pésima & PésimaCA \\
\hline E1 y E3 bajos & Dmáx grave & CA y SB mala & \\
E2 Y E3 bajos & MLI grave & BG y SB mala & MalaSB \\
E3 bajo & MLI grave & SB mala & \\
\hline E1 y E2 bajos & BLI grave & CA y BG mala & \multirow{2}{*}{ MalaBG } \\
E2 bajo & BLI grave & BG mala & \\
\hline E1 bajo & BLI grave & CA mala & MalaCA \\
\hline E1 y E3 bajos & Dmáx riesgo & CA y SB regular & \\
E2 Y E3 bajos & MLI riesgo & BG y SB regular & \\
E1 y E2 bajos & BLI riesgo & CA y BG regular & Regular \\
E3 bajo & MLI riesgo & SB regular & \\
E2 bajo & BLI riesgo & BG regular & \\
E1 bajo & BLI riesgo & CA regular & \\
\hline Ninguno bajo & Algún indicador severo, grave o en riesgo & En riesgo & Riesgo \\
Ninguno bajo & Ninguno & Adecuada & Adecuada \\
\hline
\end{tabular}

Tabla 1.

Consideraciones para el sistema de reglas de inferencia-rigidez

CA: capa asfáltica; BG: base granular; SB: sub-base 
En la figura 4 se presenta la configuración del $\mathrm{SID}_{\text {rigidez }}$ diseñado con los respectivos conjuntos difusos y funciones de pertenencia propuestos para las variables de entrada y de salida.

\section{Diseño del sistema para evaluar deterioro $\left(\mathrm{SID}_{\text {deterioro }}\right)$}

Las cuatro variables de entrada consideradas para evaluar la condición por deterioro son, las grietas, baches abiertos y baches reparados, relacionados con fenómeno de rotura del pavimento y la profundidad de roderas, asociada con problemas de deformación. Cada tipo de daño se clasifica según su intensidad, en adecuada, ligera, media y severa, cuyos límites detonan diferentes niveles de intervención; la representación difusa de estos términos y su dominio en la escala de los números reales, se definió teniendo en cuenta los criterios suge- ridos en Orozco (2005) y ARA Inc. y ERES Consultants (2004).

La salida del sistema está constituida por ocho categorías de deterioro que integran las posibles combinaciones de variables de entrada, considerando que un pavimento puede estar afectado por deformaciones, roturas o ambos. En la definición de estas categorías también se consideró su aplicación en la toma de decisiones sobre el tipo de intervención a ejecutar, pues en la práctica las acciones están sujetas a la presencia, tipo e intensidad del deterioro.

El sistema de reglas de inferencia que articula las entradas y las salidas del $\mathrm{SID}_{\text {deterioro, }}$ se estableció con base en las condiciones sintetizadas en la tabla 2.

Finalmente, las categorías de deterioro en la salida, se asociaron con una escala de calificación que varía de 0 (rotura severa) a 10 (adecuada), según se aprecia en la estructura final del SID $_{\text {deterioro }}$ diseñado, incluido en la figura 5.
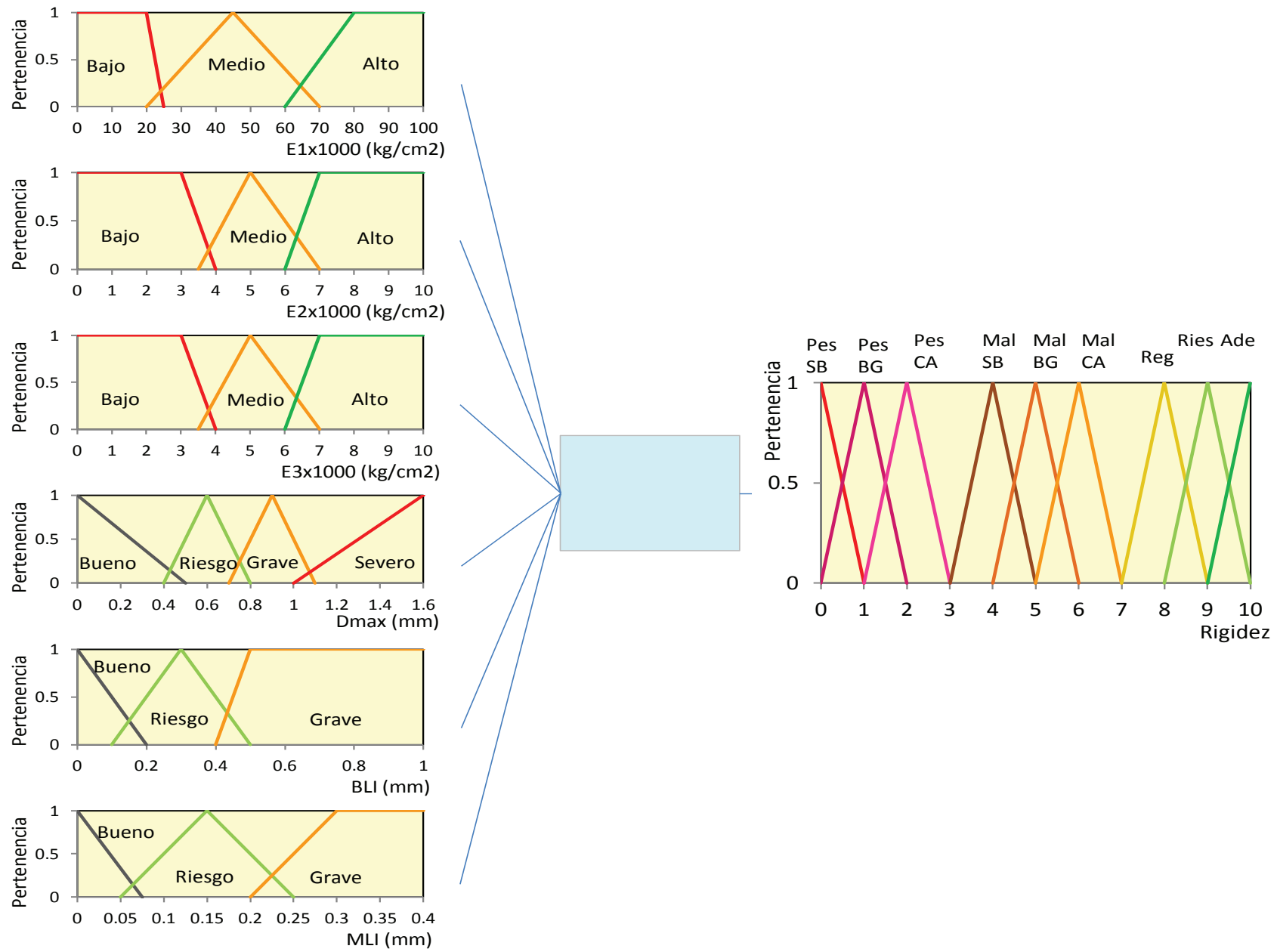

Figura 4. Sistema de inferencia para evaluar la rigidez 
Tabla 2. Consideraciones para el sistema de reglas de inferencia por deterioro

\begin{tabular}{|c|c|c|}
\hline SI & $\mathrm{O}$ & $\begin{array}{l}\text { Condición } \\
\text { por deterioro }\end{array}$ \\
\hline Grietas o Baches severos & Grietas y Baches medios & \multirow{2}{*}{ Rotura severa } \\
\hline Grietas y Roderas medias & Baches y Roderas medias & \\
\hline Grietas o Baches medios & Grietas y Baches ligeros & \multirow{4}{*}{ Rotura media } \\
\hline Grietas medias y baches ligeros & Baches medios y grietas ligeras & \\
\hline Grietas y Roderas ligeras & Baches y Roderas ligeras & \\
\hline \multicolumn{2}{|l|}{ Grietas, baches y roderas ligeras } & \\
\hline Grietas ligeras & & Grietas ligeras \\
\hline Baches ligeros & & Baches ligeros \\
\hline Roderas severas & Roderas severas y grietas ligeras & Deformación severa \\
\hline Roderas medias & Roderas medias y grietas ligeras & Deformación media \\
\hline Roderas ligeras & & Deformación ligera \\
\hline Ninguno & & Adecuada \\
\hline
\end{tabular}

Figura 5. Sistema de inferencia difuso para evaluar el deterioro
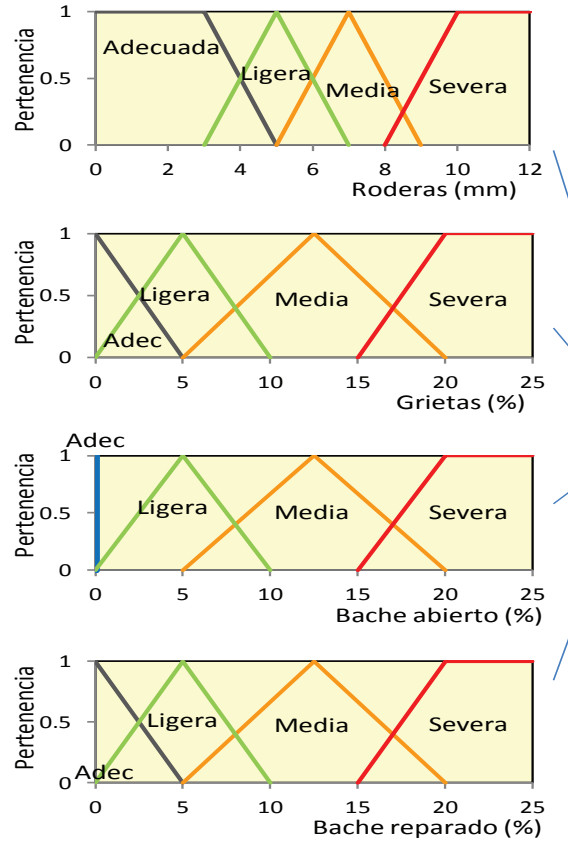

\section{Diseño del sistema para inferir las soluciones $\left(\right.$ SID $\left._{\text {soluciones }}\right)$}

En este tercer sistema se integran los criterios de rigidez y deterioro para lograr una caracterización que permita formular soluciones acordes con las dos condiciones. Se plantea que las calificaciones de salida del $\operatorname{SID}_{\text {rigidez }}$ y el $\mathrm{SID}_{\text {deterioror }}$ con sus respectivos conjuntos difusos y términos, conformen las entradas al SID soluciones.

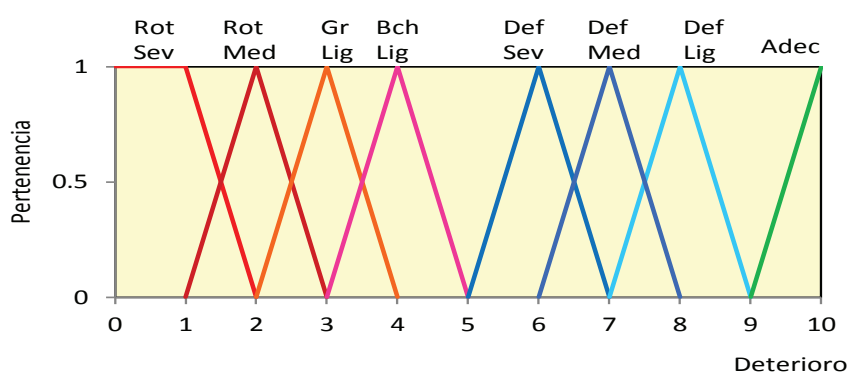

La salida del tercer sistema está constituida por una calificación en escala de 0 a 100, asociada a una condición integral y a un cierto tipo de intervención sugerido. Las calificaciones más altas representan pavimentos en excelente estado que no requieren intervención, mientras las calificaciones más bajas se asocian a las peores condiciones donde se requieren acciones de reconstrucción. 
La definición de posibles soluciones se realizó teniendo en cuenta las principales acciones contempladas en las normas N-CSV-CAR de la SCT y las recomendaciones de experiencias exitosas en conservación de pavimentos, encontradas en estudios previos a nivel local e internacional (ARA Inc. y ERES Consultants (2004); Barrantes et al., 2008; Consejo de Directores de Carreteras de Iberoamérica, 2002; Gómez et al., 2007; Orozco, 2005; U.S. Army Corps of Engineers, 2001; Zárate y Lucero, 2009).

En la tabla 4 se presenta la matriz de decisiones diseñada para generar el sistema de reglas de inferencia en función de las posibles combinaciones de rigidez y de deterioro.

Vale la pena recordar que las categorías SB, B y CA en las condiciones de rigidez pésima y mala identifican si las deficiencias afectan desde la sub-base, la base o la capa asfáltica. Asimismo, la categoría 1 en las intervenciones indica que la medida recomendada soluciona los problemas temporalmente, pero se requiere otra medida a mediano plazo.

Se recomiendan acciones de reconstrucción cuando existen simultáneamente deterioro severo y condiciones de rigidez pésima a mala; las acciones de refuerzo se plantean para las combinaciones intermedias de rigidez y deterioro y las restauraciones e intervenciones locales, para combinaciones de menor deterioro y rigidez poco comprometida. Adicionalmente, se incluye el monitoreo como medida de control, en aquellos casos donde aún no se manifiesta deterioro, pero los indicadores de rigidez reflejan alguna deficiencia. En la figura 6 se muestra el sistema de inferencia diseñado para establecer la condición estructural integral en la salida, con las respectivas categorías, calificaciones y soluciones.

\section{Evaluación de los SID diseñados, análisis de resultados}

Para cada conjunto de datos introducido en los SID, se obtuvieron en unos cuantos segundos, las calificaciones que caracterizan cada condición del pavimento con sus términos asociados.

En las figuras 7 y 8 se ilustra la variación de las calificaciones obtenidas a lo largo del corredor, representadas en escala numérica y de colores. Para efectos de visualización, se presentan las categorías globales de rigidez pésima, mala, regular; deterioro severo, medio, ligero; condición integral excelente, buena, regular, mala y pésima.

Se puede apreciar que los cambios más significativos en la calificación de rigidez, están estrechamente relacionados con los cambios derivados del tipo de estructura (tres y cuatro capas) y con la presencia de deterioro. Tales variaciones marcan diferencias de comportamiento, que resultaron útiles para identificar los ocho sectores mostrados en la figura 8; estos sectores se corroboraron con la técnica tradicional de diferencias acumuladas, propuesta por la AASHTO (1993).

Como producto final, se elaboró la tabla 5, donde se consolidan elementos importantes para tomar decisiones; se incluyen las condiciones de rigidez, de deterioro y las recomendaciones de intervención para los tramos que actualmente se encuentran afectados por algún daño.

Todas las acciones de conservación obtenidas mediante la modelación con los SID, reflejan necesidades acordes con la condición por deterioro y por rigidez que exhibe el corredor. Sectores con daños severos a medios, ameritan intervenciones mayores y aquellos

Tabla 4. Matriz de decisión para el sistema de reglas $\left(\mathrm{SID}_{\text {soluciones }}\right)$

\begin{tabular}{|c|c|c|c|c|c|c|c|c|}
\hline \multirow[b]{2}{*}{ RIGIDEZ } & \multicolumn{8}{|c|}{ DETERIORO } \\
\hline & Rotura severa & Deform. severa & Rotura media & Deform. media & Deform. ligera & Grietas ligeras & Baches ligeros & Adeacuada \\
\hline Pésima SB & \multicolumn{2}{|c|}{ Reconstrucción total (RECO TOT) } & Refuerzo y Fresado & Refuerzo + & & & & \\
\hline Pésima B & \multicolumn{2}{|c|}{ Reconstrucción parcial (RECO CA y BG) } & $\begin{array}{l}\text { + Reconstrucción a } \\
\text { mediano plazo (REF } \\
\text { Y FR1) }\end{array}$ & $\begin{array}{c}\text { Reconstrucción } \\
\text { a mediano plazo } \\
\text { (REF1) }\end{array}$ & $\begin{array}{l}\text { Microcarpeta } \\
+ \text { Refuerzo a }\end{array}$ & $\begin{array}{c}\text { Sello + } \\
\text { Refuerzo a }\end{array}$ & $\begin{array}{l}\text { Bacheo + } \\
\text { Refuerzo }\end{array}$ & \\
\hline Pésima CA & \multicolumn{2}{|c|}{ Reconstrucción superficial (RECO CA) } & & & mediano plazo & mediano plazo & a mediano & \\
\hline Mala SB & \multirow{2}{*}{\multicolumn{2}{|c|}{$\begin{array}{c}\text { Reconstrucción total (RECO TOT) } \\
\text { Reconstrucción parcial (RECO CA y BG) }\end{array}$}} & & & (MC1) & (Sello1) & plazo (Bach1) & Monitoreo \\
\hline Mala B & & & Refuerzo y Fresado & Refuerzo (REF) & & & & (MONIT) \\
\hline Mala CA & \multicolumn{2}{|c|}{ Reconstrucción superficial (RECO CA) } & & & & & & \\
\hline Regular & $\begin{array}{c}\text { Refuerzo y fresado } \\
\text { (REF y FR) }\end{array}$ & Refuerzo (REF) & $\begin{array}{l}\text { Microcarpeta y } \\
\text { fresado (MC y FR) }\end{array}$ & $\begin{array}{l}\text { Microcarpeta } \\
\text { (MC) }\end{array}$ & $\begin{array}{l}\text { Renivelación } \\
\text { local (RENI) }\end{array}$ & $\begin{array}{l}\text { Sello con o } \\
\text { sin calafateo } \\
\quad \text { (Sello) }\end{array}$ & Bacheo (Bach) & \\
\hline $\begin{array}{l}\text { Riesgo } \\
\text { Adecuada }\end{array}$ & $\begin{array}{l}\text { Microcarpeta y } \\
\text { fresado (MC y FR) }\end{array}$ & Microcarpeta (MC) & & & & & & Nada \\
\hline
\end{tabular}



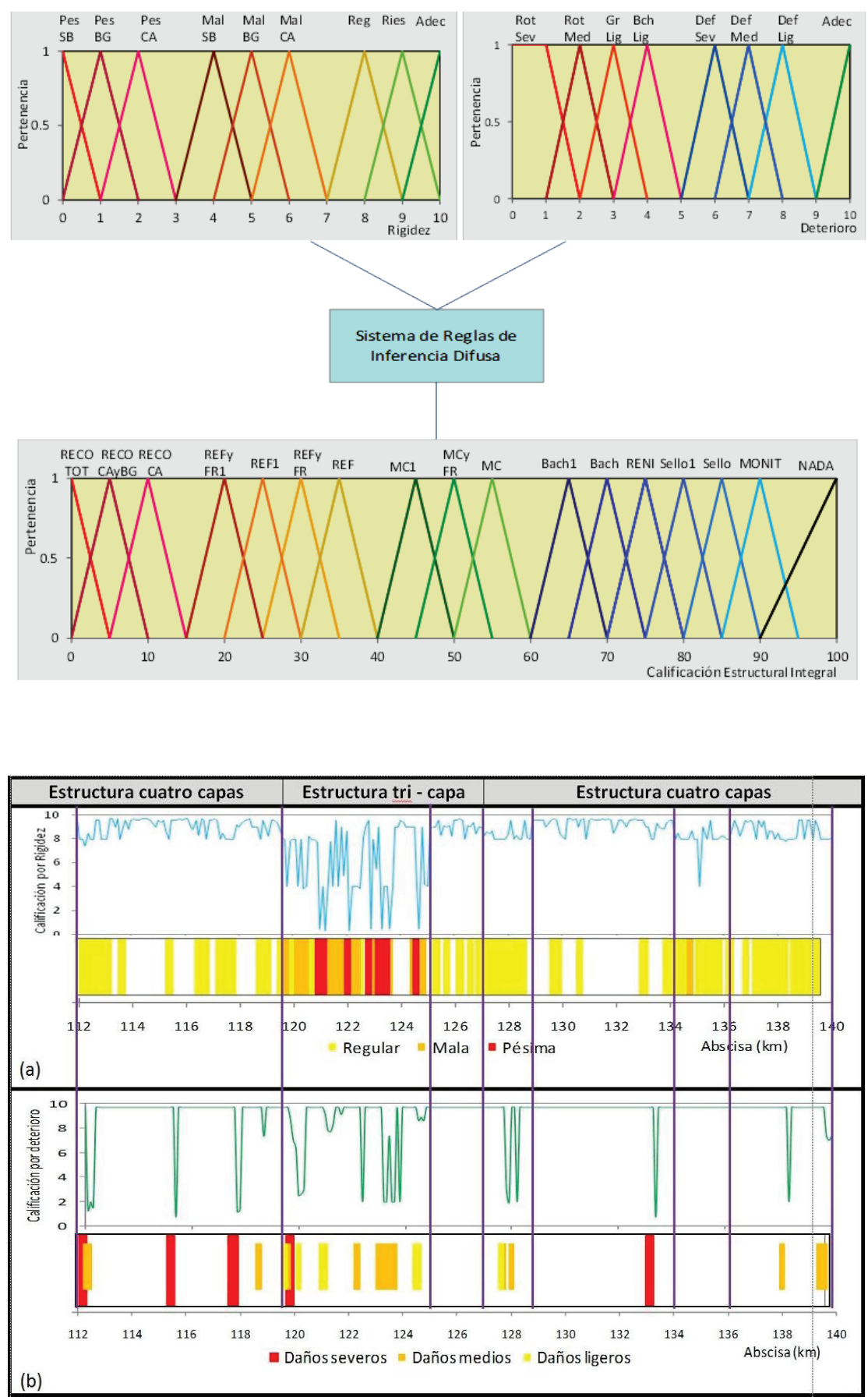

Figura 6. Sistema de inferencia difuso para la evaluación integral y soluciones

Figura 7. Caracterización del corredor (a) Según la rigidez; (b) Según el deterioro 


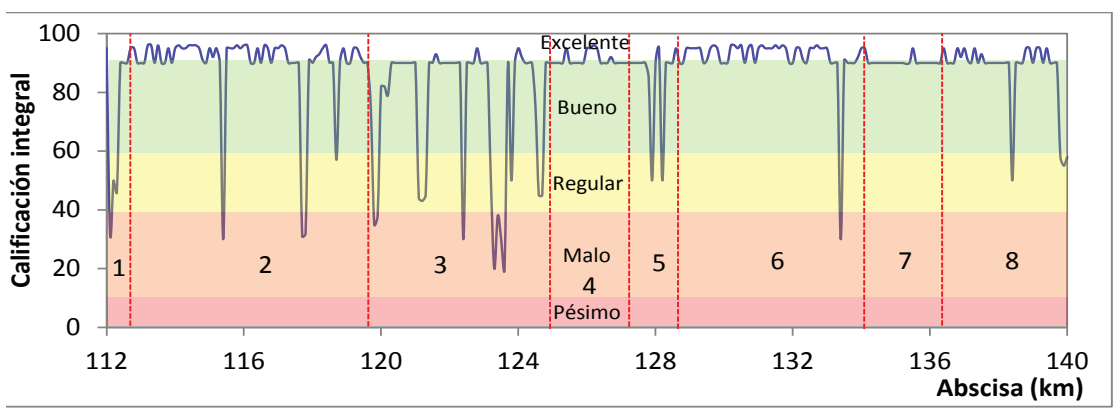

Figura 8. Caracterización de la condición estructural integral del corredor

Tabla 5. Síntesis de la evaluación estructural e intervenciones en tramos con problemas

\begin{tabular}{|c|c|c|c|c|c|c|}
\hline \multirow[b]{2}{*}{ Capas } & \multirow[b]{2}{*}{ Sector } & \multicolumn{2}{|c|}{ Subtramo } & \multirow[b]{2}{*}{ Rigidez } & \multirow[b]{2}{*}{ Deterioro } & \multirow[b]{2}{*}{ Intervención } \\
\hline & & Del Km & Al Km & & & \\
\hline \multirow{6}{*}{ Cuatro } & \multirow{3}{*}{1} & 112.1 & 112.2 & Regular & Rot. severa-media & Refuerzo y fresado, previo bacheo local \\
\hline & & 112.2 & 112.3 & Regular & Rotura media & Microcarpeta y fresado \\
\hline & & 112.3 & 112.4 & Regular & $\begin{array}{l}\text { Deform. media } \\
\text { Rotura media }\end{array}$ & Microcarpeta y refuerzo a mediano plazo $^{*}$ \\
\hline & \multirow{3}{*}{2} & 115.4 & 115.5 & \multirow{2}{*}{ Regular } & Rotura severa & Refuerzo y fresado, previo bacheo local \\
\hline & & 117.7 & 117.9 & & Rotura severa & Refuerzo y fresado, previo bacheo local \\
\hline & & 118.7 & 118.8 & En riesgo & Deforma. media & Microcarpeta \\
\hline \multirow{17}{*}{ Tres } & \multirow{17}{*}{3} & 119.7 & 119.8 & Regular & Deform. ligera & Renivelacion \\
\hline & & 119.8 & 120.0 & Mala1 & Deform. Media & Refuerzo* \\
\hline & & 119.0 & 120.0 & Regular & Rotura ligera & Neruerzo \\
\hline & & 120.0 & 120.1 & Regular & Rotura ligera & Sello \\
\hline & & 120.1 & 120.2 & Regular & $\begin{array}{l}\text { Deform. ligera } \\
\text { Rotura ligera }\end{array}$ & Sello; refuerzo a mediano plazo* \\
\hline & & 120.2 & 120.3 & Mala1 & Rotura ligera & Sello; refuerzo a mediano plazo \\
\hline & & 121.1 & 121.3 & Mala1 a pésima1 & \multirow{2}{*}{ Deforma. Ligera } & Microcarpeta; refuerzo a mediano plazo \\
\hline & & 121.3 & 121.4 & Mala1 & & Microcarpeta; refuerzo a mediano plazo \\
\hline & & 122.4 & 122.5 & Mala 1 & \multirow{2}{*}{ Rotura media } & Bacheo local, refuerzo y fresado \\
\hline & & 123.2 & 123.3 & Riesgo & & Bacheo local, microcarpeta y fresado \\
\hline & & 123.3 & 123.4 & Pésima1 & Rotura media & $\begin{array}{l}\text { Bacheo local inmediato; refuerzo y fresado; recons. } \\
\text { med. plazo }\end{array}$ \\
\hline & & 123.4 & 123.5 & \multirow{2}{*}{ Mala1 } & \multirow{2}{*}{$\begin{array}{l}\text { Deform. media } \\
\text { Rotura media }\end{array}$} & Refuerzo \\
\hline & & 123.5 & 123.6 & & & Bacheo local inmediato; refuerzo y fresado \\
\hline & & 123.6 & 123.7 & Pésima1 & \multirow[t]{2}{*}{ Rotura media } & $\begin{array}{l}\text { Bacheo local inmediato; refuerzo y fresado; recons. } \\
\text { med. plazo }\end{array}$ \\
\hline & & 123.8 & 123.9 & \multirow[b]{3}{*}{ Mala1 a pésima1 } & & Bacheo local inmediato; microcarpeta y fresado \\
\hline & & 124.5 & 124.6 & & \multirow{2}{*}{ Deform. ligera } & Renivelacion \\
\hline & & 124.6 & 124.8 & & & Microcarpeta; refuerzo a mediano plazo \\
\hline \multirow{6}{*}{ Cuatro } & & 127.8 & 127.9 & \multirow{3}{*}{ Regular } & Rotura ligera & Sello \\
\hline & 5 & 127.9 & 128.0 & & Rotura media & Bacheo local inmediato; microcarpeta y fresado \\
\hline & & 128.2 & 128.3 & & Rotura media & Bacheo local inmediato; microcarpeta y fresado \\
\hline & 6 & 133.4 & 133.5 & Regular & Rotura severa & Bacheo local inmediato; refuerzo y fresado \\
\hline & \multirow{2}{*}{8} & 138.4 & 138.5 & \multirow{2}{*}{ Regular } & Rotura media & Bacheo local inmediato; microcarpeta y fresado \\
\hline & & 139.8 & 140.0 & & Defor. Media & Microcarpeta \\
\hline
\end{tabular}

*Sectores afectados simultáneamente por rotura y deformación 
con daños ligeros requieren soluciones de restauración, a menos que la rigidez se encuentre en estado malo a pésimo.

El sector 3 (K119.7 - K125.1) requiere la mayor cantidad de acciones dadas las condiciones de rigidez y de deterioro presentes. Por el contrario, en los sectores 4 y 7 no se requiere intervención alguna, salvo por prácticas de monitoreo.

\section{Conclusiones}

Los tres sistemas planteados ofrecen la posibilidad de involucrar las variables cualitativas del pavimento y los criterios de expertos directamente en los análisis, así como orientar las recomendaciones de conservación en términos descriptivos.

Con base en los resultados obtenidos para el caso de estudio, resulta razonable pensar que la calificación lograda mediante el $\mathrm{SID}_{\text {rigidez }}$ puede utilizarse de manera confiable para definir sectores homogéneos de comportamiento.

A través del $\operatorname{SID}_{\text {rigidez }}$ y el $\operatorname{SID}_{\text {deterioro }}$ se identificaron cuatro situaciones: sectores sin problemas, sectores con daños y deficiencias de rigidez simultáneamente, sectores afectados solo por daños y sectores solo con deficiencias de rigidez; estos últimos, representan sitios que exhiben buen desempeño bajo las condiciones actuales, pero que pueden desencadenar algún deterioro en el futuro.

En general, se obtuvieron acciones de conservación acordes con la condición: en los sectores con deterioro severo a medio, el $\mathrm{SID}_{\text {soluciones }}$ asignó calificaciones asociadas con intervenciones mayores; en aquéllos con daños ligeros se plantearon soluciones de restauración, excepto donde la rigidez se caracterizó como mala a pésima. No se evidenció la necesidad de reconstrucción inmediata en ningún sector. Cabe resaltar que no existen criterios rígidos para optar por alguna de las acciones sugeridas y queda abierta la posibilidad para involucrar más soluciones en el sistema diseñado.

Gracias a la capacidad de los SID para realizar rápidamente análisis punto a punto, se proporcionan elementos para tomar decisiones globales en cada sector y particulares en sitios críticos. Esto contribuye a racionalizar las intervenciones y los recursos, en la práctica.

Ante este panorama, se considera que la lógica difusa puede jugar un rol importante tanto en el procesamiento eficiente de información, como en la modelación apropiada de sistemas estructurales de pavimento; la conveniencia o no de la aplicación de estas herramientas y el éxito que se obtenga, depende obviamente del tipo y conocimiento del problema que se quiere anali- zar. Los interesados en el uso de la herramienta presentada en este artículo, se remiten a Beltrán (2012).

\section{Referencias}

AASHTO. Guide for Design of Pavement Structures. American Association of State Highway and Transportation Officials, Washington, DC, 1993.

ARA Inc. \& ERES Consultants Division. Guide for MechanisticEmpirical Design of New and Rehabilitated Pavement Structures. Final Report, National Cooperative Highway Research Program-NCHRP, TRB, NRC, 2004.

Barrantes R., Loría L., Sibaja D. y Porras J. Desarrollo de herramientas de gestión con base en la determinación de índices para la Red Vial Nacional. Proyecto \# UI-PI-04-08. Laboratorio Nacional de Materiales y Modelos Estructurales, San José, Costa Rica, LanammeUCR. 2008.

Beltrán G. Evaluación estructural de pavimentos flexibles con métodos de inteligencia artificial y auscultación no destructiva, (tesis de doctorado en ingeniería-geotecnia), Facultad de Ingeniería, UNAM, México, DF, 2012, 166 p.

Beltrán G. y Romo M. Estimación de módulos elásticos en pavimentos usando redes neuronales artificiales, en: 14th Panamerican Conference on Soil Mechanics and Geotechnical Engineering. Toronto, Canada, 2011.

Consejo de Directores de Carreteras de Iberia e Iberoamérica, Catálogo de Deterioro de pavimentos flexibles, Colección de documentos, volumen 11, 2002

Gómez J., Pérez A. y Garnica P. Evaluación estructural de pavimentos utilizando el deflectómetro de impacto HWD (Heavy Weight Deflectometer), NOTAS núm. 108, artículo 1, Instituto Mexicano del Transporte, 2007.

Horak E. y Emery S. Falling Weight Deflectometer Bowl Parameters as Analysis Tool for Pavement Structural Evaluations, 22nd Australian Road Research Board International Conference, Australia, 2006.

Mamdani E. y Assilian S. An Experiment in Linguistic Synthesis with a Fuzzy Logic Controller. Int. J. Man-machine Studies, volumen 7, 1975: 1-13.

Orozco R. Evaluación de pavimentos flexibles con métodos no destructivos, (tesis de doctorado en ingeniería-mecánica de suelos), Facultad de Ingeniería, UNAM, México, DF, 2005, 93 p.

Sandra A., Vinayaca V., Raju K. y Sarkar A. Prioritization of Pavement Stretches Using Fuzzy MCDM Approach- a Case of Study. Soft Computing in Industrial Applications, ASC 39, 2007: 265-278.

Sundin S. y Braban-Ledoux C. Artificial Intelligence-Based Decision Support Technologies in Pavement Management, Computer-Aided Civil and Infrastructure Engineering, volumen 16 (número 2), 2001, pp. 143-157.

U.S. Army Corps of Engineers, Ladd M. y Grau R. Paver Asphalt Surfaced Airfields Pavement Condition Index-PCI. United 
Facilities Criteria-UFC-3-270-06, Department of Defense, US, Department Research and Development Center, Vicksburg, MS, 2001.

Williams R., Adballah I. y Nazarian S. Implementation of Data Fusion Techniques in Non Destructive Testing of Pavement. Research Report 0-4393-2; Research Project 0-4393. Center for Transportation Infrastructure Systems, The University of Texas at El Paso, TX, Texas Department of Transportation \& Federal Highway Administration, 2004.

Yella S., Dougherty M. y Gupta N. Artificial Intelligence Techniques for the Automatic Interpretation of Data from Non-Destructive Testing, Non-Destructive Testing and Condition Monitoring. Insight, volumen 48, (número 1), 2006: 10-20.

Zadeh L. Fuzzy Sets. Information and Control, volumen 8, 1965: 338-353.

Zárate M. y Lucero M. Análisis de la respuesta de pavimentos flexibles, utilizando el deflectómetro HWD, Sexto Congreso Mexicano del Asfalto. Revista técnica asfáltica (número 19), 2009.

\section{Este artículo se cita:}

\section{Citación estilo Chicago}

Beltrán-Calvo, Gloria Inés, Miguel Pedro Romo-Organista. Evaluación de pavimentos y decisiones de conservación con base en sistemas de inferencia difusos. Ingeniería Investigación y Tecnología, XV, 03 (2014): 391-402.

\section{Citación estilo ISO 690}

Beltrán-Calvo G.I., Romo-Organista M.P. Evaluación de pavimentos y decisiones de conservación con base en sistemas de inferencia difusos. Ingeniería Investigación y Tecnología, volumen XV (número 3), julio-septiembre 2014: 391-402.

\section{Semblanza de los autores}

Gloria Inés Beltrán-Calvo: Es ingeniera civil (1991) y magíster en geotecnia (1999) por la Universidad Nacional de Colombia; doctora en ingeniería por la Universidad Nacional Autónoma de México (2012). Es profesora Asociada de la Facultad de Ingeniería de la Universidad de Colombia desde el año 2000, donde tuvo a cargo el laboratorio de suelos, rocas y pavimentos en el periodo 2000-2007. Sus áreas de interés e investigación son: gestión de la infraestructura vial, materiales para carreteras, petrografía aplicada a la evaluación de mezclas asfálticas y agregados pétreos, métodos de análisis basados en herramientas de la inteligencia artificial, en especial, redes neuronales artificiales y lógica difusa.

Miguel Pedro Romo-Organista: Es ingeniero civil por la Universidad Autónoma de Guadalajara (1968). Obtuvo la maestría en mecánica de suelos por la Universidad Nacional Autónoma de México, UNAM (1972) y el doctorado en ingeniería geotécnica por la Universidad de California, Berkeley en 1976. Es profesor investigador titular del Instituto de Ingeniería, UNAM desde 1977. Obtuvo el Premio Nacional de Ciencias y Artes 2007 por la Presidencia de la República, el Premio Universidad Nacional 2005 en el área de Innovación Tecnológica y Diseño Industrial, UNAM y el Premio Nabor Carrillo Flores 2004-2005, Colegio de Ingenieros Civiles de México. Excellent Contributions Award, International Association for Computer Methods and Advances in Geomechanics, 2005. Ha dirigido 15 tesis de doctorado, 33 de maestría y 9 de licenciatura y es investigador nacional emérito, Sistema Nacional de Investigadores. 